\title{
Field Effect Transistors based on Polycyclic Aromatic Hydrocarbons for the Detection and Classification of Volatile Organic Compounds in a Background of Humidity
}

\author{
A.Bayn ${ }^{l}$, R.Ionescu ${ }^{2}$, H.Hossam ${ }^{l}$ \\ ${ }^{I}$ Dept. of Chemical Eng., Technion - Israel Institute of Technology, Haifa 3200003, Israel.,
}

\author{
Lila1eye@gmail.com
}

${ }^{2}$ Dept. of Electronics, Electrical and Automatic Eng., Rovira i Virgili Univ., 43007 Tarragona, Spain.

\begin{abstract}
:
Polycyclic aromatic hydrocarbon (PAH) based field effect transistor (FET) arrays show great promise in the field of sensors, detecting various chemical species. Nevertheless the discrimination abilities of the FET PAH sensors as an E-nose application have not been fully investigated. In present work we introduce silane modified PAH based sensors, capable of withstanding the confounding humidity conditions, we conduct an analysis of the various electrical features, characterizing field effect transistors, of 4 different PAH coated devices. Using appropriate statistical analysis we demonstrate the discrimination abilities of the array between polar, non-polar and aromatic chemicals and the advantages of using FET devices. The ability to study different electrical features may perhaps hold the key for better understanding the relationship between the sensor and the analyte and more importantly may bring us one step closer to creating a unique fingerprint to each analyte.
\end{abstract}

Key words: Polycyclic aromatic hydrocarbon, sensor, volatile organic compound, humidity, field effect transistor.

\section{Introduction}

We present a technology based on an array of Field Effect Transistors (FETs) coated with a layer of different (semi-)conducting PAHs. Our study is based on the fact that PAH molecules are able to self-assemble into molecular stacks with large, electron-rich, semiconducting core, which guarantees good charge carrier transport along the molecular stacking direction. With this architecture, we investigate the possibility of using PAH-FETs as fast, non-invasive portable technology that can be used for a widespread detection of various VOCs. We investigate optimal ways to utilize independent electrical parameters (e.g., voltage threshold, mobility, on/off ratio) of PAH-FETs on the classification of various VOCs and humidity backgrounds. Amongst the rest, we: (i) investigate the usage of a combination of different electrical parameters, extracted from a single PAH-FET, as an array of virtual sensors; and (ii) investigate the possibility of using a combination of different electrical parameters, extracted from an array of various PAH-FETs, as an array of virtual sensors. The relation between the extracted electrical parameters and the classification accuracies of VOCs is presented and discussed.

\section{Experimental}

TheFET devices were fabricated on p-type $\mathrm{Si}(100)$ wafer covered with a $300 \mathrm{~nm}$ thick thermally grown $\mathrm{SiO} 2$ insulating layer. The surface of the FET's SiO2 layer was functionalized with a Hexyltrichlorosilane (HTS) monolayer. Previous works showed that 
adsorption of HTS monolayer on a (native) $\mathrm{SiO} 2 / \mathrm{Si}$ surface improves both electrical and sensing properties of the FET device.[1-6]The HTS-terminated $\mathrm{SiO} 2$ surface was coated $\mathrm{PAH}$ film that was chosen from a reservoir of four $\mathrm{PAH}$ derivatives, which differfrom each other in

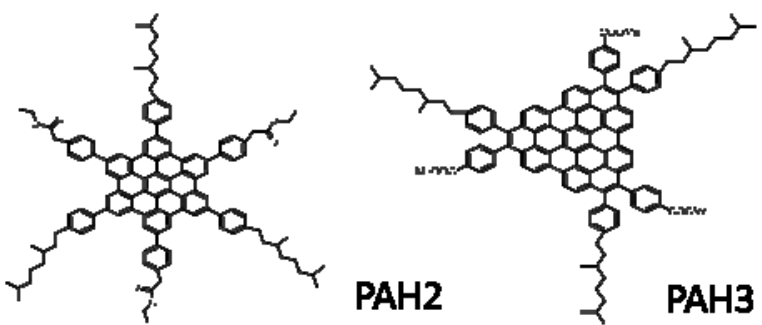

Fig. 1: Chemical structure of the PAH derivatives.

\section{Silane modification}

One of the major obstacles to use FET as a gas sensor is linked with the hysteresis effect. The silicon oxide (SiO2) surface allows the formation of surface trap states $\left(\mathrm{Si}-\mathrm{OH}, \mathrm{Si}-\mathrm{O}^{-}\right.$, $\mathrm{Si}-\mathrm{OH} 2+$ species) that can function as sorption sites and, therefore, capture water molecules. When the relative humidity of the environment is high enough, water drops condensate on the $\mathrm{SiO} 2$ surface and become ionized. The result is strong changes in the FET characteristics that mask the mild changes in current induced by the targeted analyte(s).[7]The $\mathrm{SiO} 2$ surface is extremely relevant to our case since the coverage area of the different $\mathrm{PAH}$ derivatives is not always $100 \%$.

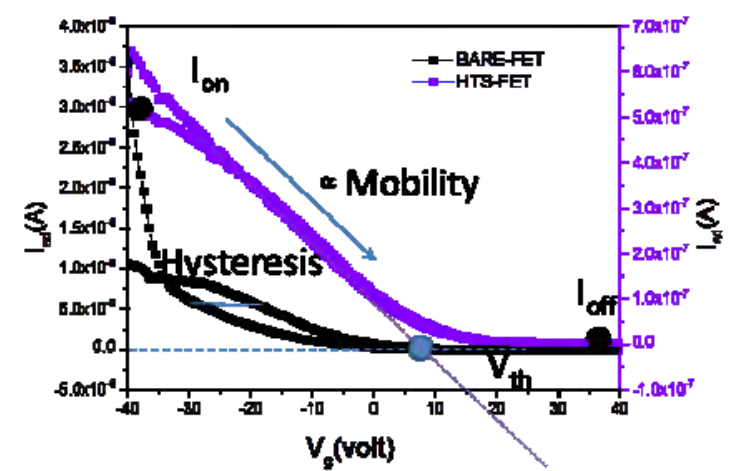

Figure 2. Linear characteristics of the source-drain current (Isd) versus gate voltage (Vg) of FET covered in $\mathrm{PAH}-2$ layer with (purple curve) and without (black curve) HTS layer at the interface. The measurements were carried out by a forward and backward scan of $\mathrm{Vg}$ steps of $500 \mathrm{mV}$, at $\mathrm{Vsd}=30 \mathrm{~V}$.

As a way to reduce the hysteresis effect, we have chosen to modify the $\mathrm{SiO} 2$ surface with hexyltrichlorosilane (HTS) molecules. This process has been proven as an efficient way to reduce the percentage of the tap states (mainly, either the aromatic core or/and the side chains$\mathrm{PAH}-2, \mathrm{PAH}-3, \mathrm{PAH}-4$ and $\mathrm{PAH}-7$ (see figure 1). Electrical measurements were made before and after exposure to a series of polar and nonpolar VOCs under various humidity backgrounds.
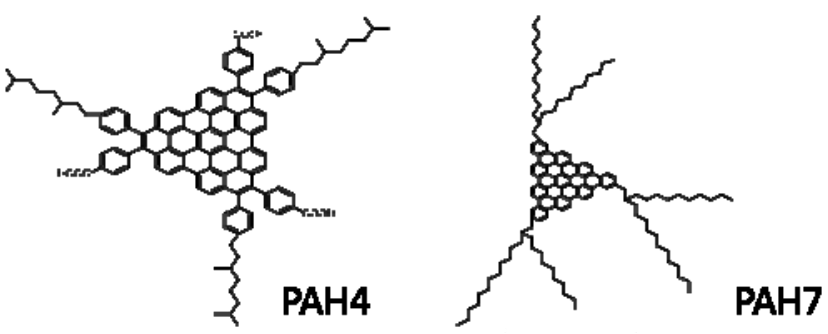

$\mathrm{Si}-\mathrm{OH}$ groups) on the $\mathrm{SiO} 2$ surface, thus enhancing the related electrical and sensing features of the device[8][9] - NOTE: only the $\mathrm{SiO} 2$ part of the FET is covered with the HTS molecules, leaving a direct contact between the

semiconducting $\mathrm{PAH}$ layer and the source and drain metal electrodes. The HTS surface modificationimproved almost all electrical features of the studied PAH-FETs, all sensors have been studied in the same manner seen in figure 2. The main improvement is the clear effect on the hysteresis of the examined devices, in the following order: $\mathrm{PAH}-2<\mathrm{PAH}-4$ $<\mathrm{PAH}-3<\mathrm{PAH}-7$. These results indicate that the PAH-2 with HTS modification has shown the largest decrease in hysteresis and is the least sensitive to water.

The various sensors were exposed to varying environments of analyte flow (20 min) than air flow(30 $\mathrm{min})$.In each step the gate voltage $(\mathrm{Vg})$ applied was changed between $-40 \mathrm{~V}$ and $+40 \mathrm{~V}$ with $500 \mathrm{mV}$ steps and at $30 \mathrm{~V}$ source-drain voltage(Vds). In every step the following features were extracted: (1) $1 @ V_{g}=-20 \mathrm{~V}$, the current value at gate voltage of $-20 \mathrm{~V}$. (2) $\mu_{\mathrm{h}}$, hole mobility values extracted from the $I_{d s} v s . V_{g}$ curve. (3) $V_{\text {th }}$, voltage threshold calculated by extrapolating the linear part of the $I_{d s} v s . V_{g s}$ curve and extracting the interception value with the voltage axis. (4) $I_{\text {on/off }}$, the ration between the current-on $\left(\mathrm{I}_{\mathrm{on}}\right)$, and current-off $\left(\mathrm{I}_{\mathrm{off}}\right)$, calculated by dividing the current value when the device is "open" (i.e., $\mathrm{I}_{\text {on }}$ )- an average of five measurements of current ending with $V_{g}=-40 \mathrm{~V}$, by the value when the device is "closed" (i.e., $\mathrm{I}_{\text {off }}$ )- an average of five measurements of current starting with $\mathrm{V}_{\mathrm{g}}=+40 \mathrm{~V}$. The value of each electrical feature $\left(I @ V_{g}=-20 V, \mu, V_{\text {th }}\right.$, lon/off) was plotted as a function of time before, during, and after each exposure step.Normalized values of the feature were 
extracted, meaning, the change in the sensor's steady-state conductivity, averaged over 5 measurements at the end of the VOC exposure, compared to the average of 5 measurements at the end of the baseline (exposure to dry air) and divided by the baseline value.Finally the four normalized features used in the following analysis are: (1) $\Delta \mathrm{I} / \mathrm{I}_{0}$, normalized current (at $\mathrm{Vg}=-20 \mathrm{~V}) ; \quad(2) \Delta \mu / \mu_{0}$, normalized mobility; (3) $\Delta \mathrm{V}_{\mathrm{th}} / \mathrm{V}_{\mathrm{th}}$, normalized voltage threshold;

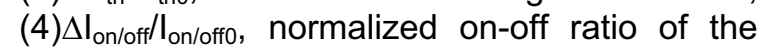
current.

\section{Sensing measurements}

Figure 3 presents the normalized mobility values of the sensors, when exposed to various VOCs, in the same manner the three other normalized features were analyzed. As seen in the figure, most of the exposures result in a decrease in normalized mobility. $\mathrm{PAH}-2$ and $\mathrm{PAH}-3$ exhibited only a decrease in mobility in both low (5\%) and high (40\%) relative humidity $(\mathrm{RH})$ environments. On the other hand, $\mathrm{PAH}-4$ and $\mathrm{PAH}-7$ showed both increase and decrease, depending on the types of the exposed VOC and $\mathrm{RH}$ level. $\mathrm{PAH}-2$ and $\mathrm{PAH}-3$ exhibit high discrimination ability between the various VOCs based on the normalized mobility feature alone. $\mathrm{PAH}-2$ is of special interest since the discrimination ability of this device was not (or minimally) affected by the $\mathrm{RH}$ level of the exposure environment. Indeed, neither the response magnitude nor the ratio between the responses obtained upon exposure towards various VOCs was affected. The substantial decrease in normalized mobility upon exposure to aromatic VOCs could be related to one or both of the following reasons: (i) the aromatic VOCs have higher bonding strength to the surface, causing greater interruption to the charge carrier's mobility, perhaps due to swelling effect; (ii) the higher absorption ability of aromatic VOCs causes a greater dipoleinduced field than the other groups of VOCs (alcohol and alkane), interfering with the field caused by the gate electrode. No relationship was found between the normalized mobility response and the polarity of non-aromatic VOCs.PAH-3 and $\mathrm{PAH}-4$ have the same aromatic core (semi-triangle) and organic side chains, but slightly differ in the termination groups of the organic side chains (ester group in the PAH-3 and carboxylic group in the PAH4). Integrating the $\mathrm{PAH}-3$ and $\mathrm{PAH}-4$ in FET platforms exhibited substantially different normalized mobility responses upon exposure to the same conditions. This observation stresses the importance of the PAH's side chain for sensing applications. Side chains mediate the interaction between the VOC and the charge carrier channel of the organic semiconductor. They also play an important role in the self-assembly and morphology of the $\mathrm{PAH}$ on the surface, both affecting the sensing mechanism.

When examining the values of the various features, we can see that each feature of each sensor has its own behavior as a result of the exposure, varying from analyte to analyte and based on humidity levels. These variations of features and sensors give us the variability we need in the array of sensors as a hole, to discriminate between the analytes exposed.

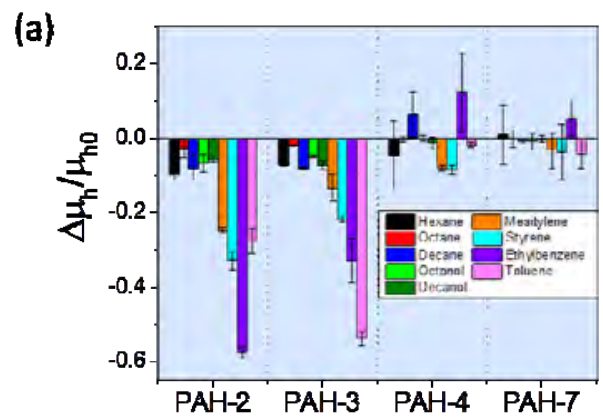

(b)

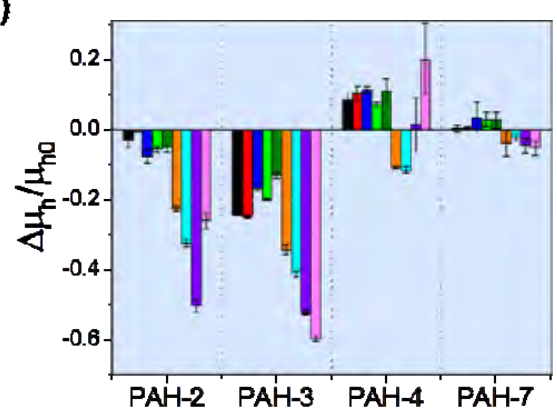

$\mathrm{Fi}$

gure 3. Normalized mobility values while exposing the devices studied to different analytes in an environment of (a) $5 \% \mathrm{RH}$; and (b) $40 \% \mathrm{RH}$. The concentration in both figures is $P / P O=0.1$. Each value is the average of 2 repetition of exposure in the same conditions.

\section{Discriminant Factor analysis}

The normalized feature valuesgive us a great amount of data, each sensor and each feature have their own response to the exposures and a pattern in most cases is difficult to observe. In order to focus our results to a productive way of discrimination between the VOCs, we employed a discriminant factor analysis (DFA) algorithm.[10] DFA is a supervised linear method that is supplied with the classification information regarding every measurement in the training set. The DFA model is built in order to classify a set of predefined groups, with the help of input variables (normalizes features) for the members of the known groups. The accuracy of DFA was further confirmed by 
employing leave-one-out cross-validation. The DFA algorithm was applied on the normalized features extracted from the chosen sensors in three different ways: (i) separating between the aromatic and non-aromatic VOCs (ii) separating polar and non-polar analytes (iii) separating each of the sub-groups to its composing analytes.

Figure 4 presents the DFA results of the separation of the aromatic and non-aromatic VOC groups. A high separation accuracy (using the leave one out method) of $95.37 \%$ is received using only one variable- $\Delta \mu / \mu_{0}$ of $\mathrm{PAH}-$ 2 sensor.

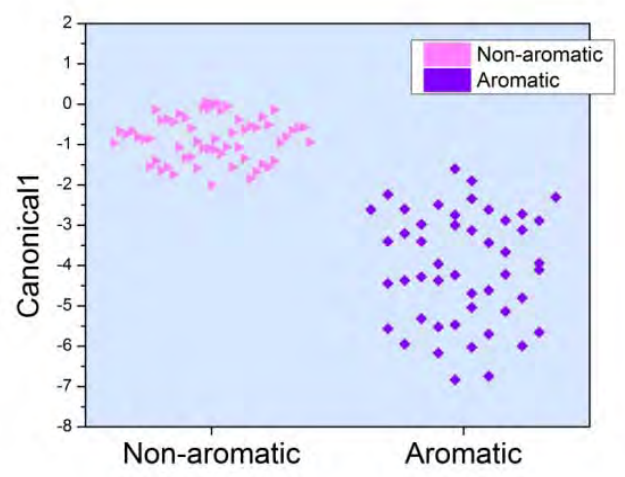

Figure 4. DFA results separating the non-aromatic (octanol, decanol, hexane, octane, decane) and aromatic analytes (mesitylene, styrene, ethylbenzene, toluene). The different dots stand for the response of the device upon exposure to different concentrations $(p / p o=0.05,0.1,0.2)$ of the different VOCs in both $5 \%$ and $40 \% \mathrm{RH}$. Each exposure has two repetitions.

A single sensor provides us with a high accuracy separation, despite the fact that the analytes are tested in different humidity conditions and different concentrations. The fact that the separation is based on PAH-2 matches the conclusions from figure 2, which shows that $\mathrm{PAH}-2$ is the least sensitive to water/humidity. $\mathrm{PAH}-2$ makes it possible to separate between analytes according to their chemical or physical properties, rather than according to the confounding humidity. The choice of the normalized mobility feature is also not coincidental, we can see from Figure 3, the normalized mobility of PAH2 gives significantly different values when exposed to aromatic analyte compare to non-aromatic ones and less-effected by the humidity change. Decreasing our array to one sensor holds a great significance, making the final device easy to use, the results more simply analyzed and production issues minimized.

Once we are able to separate the analytes into two groups or aromatic (non- aromatic) we can proceed to the separation of the non-aromatic group into polar and non-polar analytes, the results are presented in Figure 5 . We use, in present seperation 8 different combinations of the four PAHs and three electrical features in the following manner: $\Delta \mathrm{l} / \mathrm{l}_{0}$ of $\mathrm{PAH} 2,3$, $4 \& 7, \Delta \mu / \mu_{0}$ of $\mathrm{PAH} 4 \& 7$ and $\Delta \mathrm{Vth} / \mathrm{Vth} \mathrm{th}_{0}$ of $\mathrm{PAH}$ $3 \& 7$. The accuracy of separation received by the leave on out method is $80 \%$.

We can clearly see from the results that the array is less sensitive to this particular separation.We use all the sensors and all the different type of electrical feature and receive a lower accuracy than the previous one.

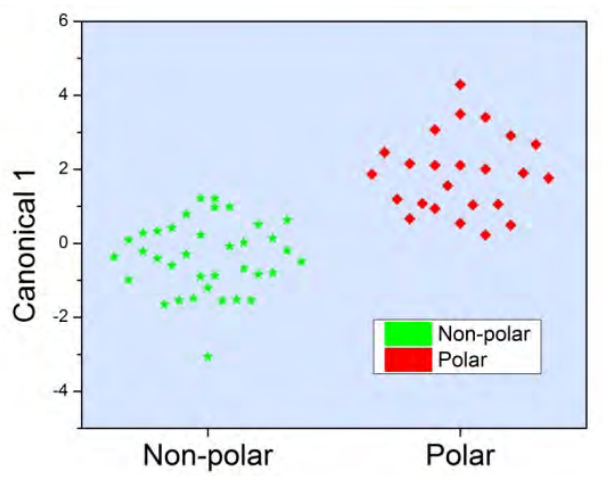

Figure 5.DFA results separating the polar material (octanol, decanol) from the non-polar (hexane, octane, decane). The different dots stand for the response of the device upon exposure to different concentrations $(\mathrm{p} / \mathrm{p} 0=0.05,0.1,0.2)$ of the different VOC's in both $5 \%$ and $40 \%$ RH. Each exposure has two repetitions.

Using a combination of all sensors and all features provides better results than using some of the sensors or some of the features (results not presented here). The interaction between the analyte and the sensor is restricted to the mechanism between them, thus using only one electrical feature (as done in arrays of chemiresistors for example) limits the discrimination abilities of each sensor and the array of sensors as a hole. A FET array allows us to extract several features while choosing the most suitable one, according to our separation needs. Although we are limited by only 4 sensors in the array we can still receive separation by choosing the most suitable combination of features from each sensor.

Now that we were able to separate the polar and non-polar groups, we want to test the arrays ability to differentiate between the analytes in each group. In the aromatic group (see figure 6a) the separation is based on 8 parameters $-\Delta \mathrm{l} / \mathrm{I}_{0}$ of $\mathrm{PAH} 2 \& 7, \Delta \mathrm{Vth} / \mathrm{Vth} \mathrm{th}_{0}$ of $\mathrm{PAH}$ $2 \& 7$ and $\Delta \mu / \mu_{0}$ of $\mathrm{PAH} 2 \& 3 \& 4 \& 7$, the received accuracy is $75 \%$. All the sensors and all the features were needed for the separation.We 
can assume that the high absorbance of the aromatic analytes as appose to the other analytes makes them easily discriminated, but classifying each aromatic analyte is more difficult to achieve.Looking at the figure we can see that the analytes are separated on the canonical-1 axis according to their polarity (except for ethyl benzene). The least polar analyte- mesitylene is on the right and toluene (most polar) on the left.No clear separation can be seen between the $5 \% \mathrm{RH}$ and the $40 \%$ of the same analyte, being an indication that the separation is less sensitive to humidity changes.
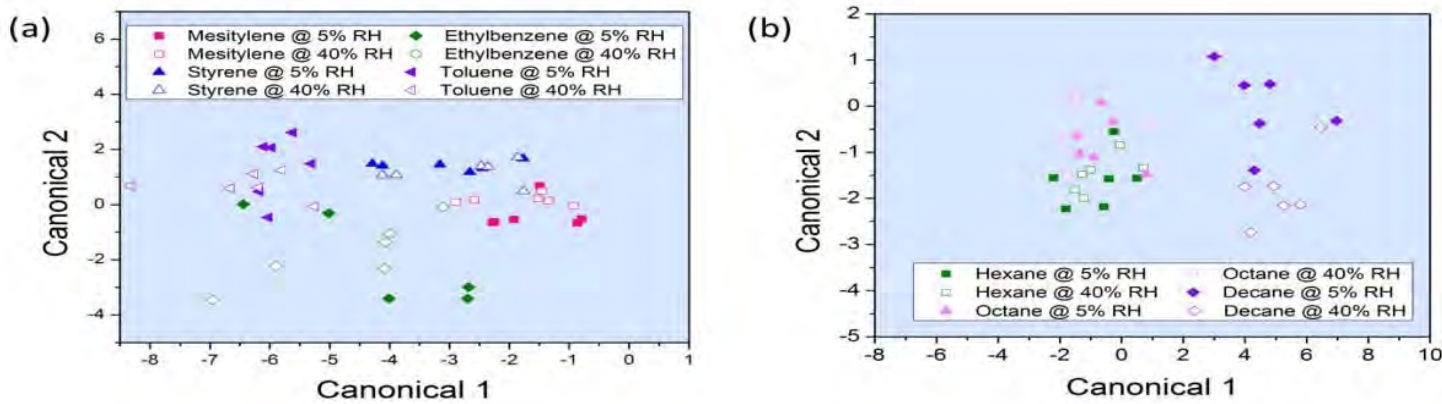

(c)

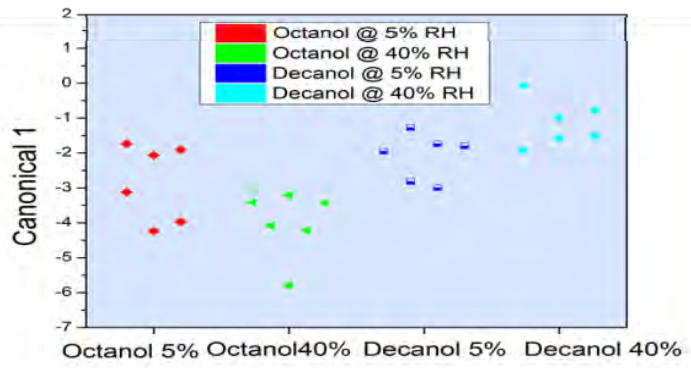

Figure 6. DFA results separating (a) aromatic material (mesitylene, styrene, ethyl-benzene, toluene); (b) non-polar VOC's (hexane, octane, decane); (c) polar materials (octanol, decanol). The different dots stand for the response of the device upon exposure to different concentrations ( $p / p 0=0.05,0.1,0.2)$ of the different VOC's in both $5 \%$ and $40 \% \mathrm{RH}$. Each exposure has two repetitions.

Figure $6 \mathrm{~b}$ presents the separation analysis between the different VOCs in the non-polar group (hexane, octane and decane). The obtained accuracy was $75 \%$ and is based on 6 parameters $\Delta \mathrm{l} / \mathrm{I}_{0}$ of $\mathrm{PAH} 3 \& 7, \Delta \mathrm{Vth} / \mathrm{Vth} \mathrm{th}_{0}$ of $\mathrm{PAH}$ $3 \& 7$ and $\Delta \mu / \mu_{0}$ of $\mathrm{PAH} 3 \& 4$. According to the figure decane is separated from the rest of the analytes on the canonical 1 axis while hexane and octane are separated by the canonical 2 axis. Here as before no significant difference can be noticed between the $5 \% \mathrm{RH}$ and the $40 \% \mathrm{RH}$. The combination of variables does not use all the sensors (no PAH-2) but includes all features to receive an accuracy of $75 \%$. The same accuracy can be achieved by using all the sensors in a different combinations not presented here. By including more features in the analysis we can reduce, in this case, the number of sensors in the array and still receive the same accuracy. Using less sensors but extracting more features of the sensors that give us more information regarding the specific separation wanted can providesame results.

Instead of including another kind of device to improve the sensing abilities of the array we can use another feature of the same sensor.
Figure $6 \mathrm{c}$ presents the separation of the polar group (between octanol and decanol); analysis is based on 4 parameters: $\Delta \mathrm{I} / \mathrm{I}_{0}$ of $\mathrm{PAH} 3 \& 4$ and $\Delta \mu / \mu_{0}$ of $\mathrm{PAH} 2 \& 3$, the accuracy of separation $79.17 \%$. We can see from the figure that the two analytes are separated on the canonical-1 axis. As appose to analyzing non-polar VOCs (where PAH-3\&4\&7 were used) in the polar VOC analysis we substitute PAH 7 (non-polaraliphatic side group) with $\mathrm{PAH}-2$ (polar -ester side group), emphasizing the fact that controlling the side groups, controls the adsorption of the VOCs thus affecting the sensing abilities. We can notice that our array is more sensitive to polar analytes $(79.17 \%$ accuracy of separation) than non-polar ones (75\% accuracy of separation) this is perhaps due to the fact that most of the sensors have polar side groups screening the absorption of the non-polar analytes. In the separation of the polar analytes the highest accuracy established with a combination of variables that do not contain all the sensors and not all the features.

\section{Conclusions}

We presented in this study a characterization of the PAH, silane modified, array of sensors, its capabilities, advantages and possible 
applications. The silane modification lowered the hysteresis affect and increased the electrical field effect features of the sensors, this was evident from the $\mathrm{PAH}-2$ response being the least sensitive to water due to the low hysteresis effect after modification, making it the main contributor in some of the DFA studies. After extracting the different features from the sensors we used the DFA method and deducted several conclusions:

(1) DFA studies showed that the aromatic analytes can be accurately separated from the rest of the analytes using only one sensor with one feature. All sensors react strongly to the exposure of the aromatic VOCs, most likely due to the similar aromatic rings as the cores of the $\mathrm{PAH}$ derivatives, making a $\pi-\Pi$ connection. Nevertheless, one sensor is enough to make the discrimination and if that's the only separation needed the application becomes extremely efficient and cost effective.

(2) When different separations are needed for example the separating the nonpolar VOCs one by one, a combination of sensors and electrical features are used. The use of an array of field effect transistor sensors increased the sensing abilities than that of the individual sensor. Each feature reacts differently to the changing environment making it a sensor of its own.

An important advantage became clear from the study-the ability to select the feature that suits us most. Whether we want a separation sensitive to different humidity environments, sensitive to a group of analytes or to specific analytes within a certain chemical group, we can choose the most suitable feature or a combination of several features.

In present study we examined only four electrical features of each sensor, but there is more information that could by extracted in the form of other features such as- normalized sub threshold swing response, multiple normalized current responses in different gate voltages and so on. The ability to study different electrical features of the sensors may perhaps hold the key for better understanding the relationship between the sensor and the analyte and more importantly may bring us one step closer to creating a unique fingerprint to each analyte.

\section{References}

1.

Paska, Y. and H. Haick, Controlling Surface Energetics of Silicon by Intermolecular Interactions between Parallel Self-
Assembled Molecular Dipoles. J. Phys. Chem. C, 2009. 113: p. 1993.

2. Paska, Y. and H. Haick, Controlling properties of field effect transistors by intermolecular crosslinking of molecular dipoles. Appl. Phys. Lett., 2009. 95: p. 233103/1.

3. Paska, Y. and H. Haick, Systematic crosslinking changes within a self-assembled monolayer in a nanogap junction: A tool for investigating the intermolecular electronic coupling. J. Am. Chem. Soc., 2010. 132: p. 1774-1775

4. Paska, Y. and H. Haick, Interactive effect of hysteresis and surface chemistry on gated silicon nanowire gas sensors. ACS Appl. Mater. Interf., 2012. 4: p. 2604-2617.

5. Paska, Y., et al., Molecular Gating of Silicon Nanowire Field-Effect Transistors with Nonpolar Analytes. ACS Nano, 2012. 6: p. 335-345.

6. Paska, Y., et al., Enhanced Sensing of Nonpolar Volatile Organic Compounds by Silicon Nanowire Field Effect Transistors. ACS Nano, 2011. 5: p. 5620-5626.

7. Tripp, C. and M. Hair, Reaction of alkylchlorosilanes with silica at the solid/gas and solid/liquid interface. Langmuir, 1992. 8: p. 1961-1967.

8. Paska, Y. and H. Haick, Interactive Effect of Hysteresis and Surface Chemistry on Gated Silicon Nanowire Gas Sensors. ACS Applied Materials \& Interfaces, 2012. 4(5): p. 2604-2617.

9. Paska, Y. and H. Haick, Controlling properties of field effect transistors by intermolecular crosslinking of molecular dipoles. Appl. Phys. Lett., 2009. 95: p. 233103/1.

10. Brereton, R.G., Chemometrics, Application of Mathematics and Statistics to Laboratory Systems. Ellis Horwood: Chichester, 1990. 